\title{
Development and clinical utility of a novel diagnostic nystagmus gene panel using targeted next-generation sequencing
}

\author{
Mervyn G Thomas ${ }^{\star, 1,2}$, Gail DE Maconachie ${ }^{1,2}$, Viral Sheth ${ }^{1}$, Rebecca J McLean ${ }^{1}$ and Irene Gottlob ${ }^{\star, 1}$
}

Infantile nystagmus (IN) is a genetically heterogeneous disorder arising from variants of genes expressed within the developing retina and brain. IN presents a diagnostic challenge and patients often undergo numerous investigations. We aimed to develop and assess the utility of a next-generation sequencing (NGS) panel to enhance the diagnosis of IN. We identified 336 genes associated with IN from the literature and OMIM. NimbleGen Human custom array was used to enrich the target genes and sequencing was performed using HiSeq2000. Using reference genome material (NA12878), we show the sensitivity (98.5\%) and specificity (99.9\%) of the panel. Fifteen patients with familial IN were sequenced using the panel. Two authors were masked to the clinical diagnosis. We identified variants in $12 / 15$ patients in the following genes: FRMD7 $(n=3)$, CACNA1F $(n=2), \operatorname{TYR}(n=5)$, CRYBA1 $(n=1)$ and TYRP1 $(n=1)$. In 9/12 patients, the clinical diagnosis was consistent with the genetic diagnosis. In 3/12 patients, the results from the genetic diagnoses (TYR, CRYBA1 and TYRP1 variants) enabled revision of clinical diagnoses. In 3/15 patients, we were unable to determine a genetic diagnosis. In one patient, copy number variation analysis revealed a FRMD7 deletion. This is the first study establishing the clinical utility of a diagnostic NGS panel for IN. We show that the panel has high sensitivity and specificity. The genetic information from the panel will lead to personalised diagnosis and management of IN and enable accurate genetic counselling. This will allow development of a new clinical care pathway for IN.

European Journal of Human Genetics (2017) 25, 725-734; doi:10.1038/ejhg.2017.44; published online 5 April 2017

\section{INTRODUCTION}

Infantile nystagmus (IN) is characterised by the involuntary oscillation of the eyes present at birth or manifesting during infancy. Nystagmus has an estimated prevalence of 2.4 in $1000^{1}$ and is associated with significant negative social stigma and poor visual function scores. ${ }^{2,3}$ IN is a genetically heterogeneous disorder that can arise due to variants of genes involved in retinal and brain development. Identifying the underlying cause of IN can be challenging due to the diverse range of genetic disorders associated with it. For example, variants that affect the development of retinal photoreceptors result in achromatopsia, congenital stationary night blindness or retinal dystrophies, all of which are typically associated with IN. ${ }^{4,5}$ Similarly, variants that cause albinism, Hermansky Pudlak syndrome and aniridia can also present with foveal hypoplasia and IN. ${ }^{5,6}$ Recently it has been shown that a small proportion of patients with FRMD7 variants can have foveal hypoplasia and IN. ${ }^{7}$ Similarly, recessive variants of SLC38A8 are associated with foveal hypoplasia. ${ }^{8}$ Congenital neurological syndromes such as spinocerebellar ataxia, episodic ataxia and structural malformations (eg microcephaly) can also present with IN, however, typically with distinctive features such as cerebellar signs. ${ }^{9}$ Often there is significant overlap in phenotypical characteristics between these disorders. This results in a long differential diagnosis list based on an often challenging clinical examination of a child with nystagmus.
Patients with IN often undergo numerous investigations to identify the underlying cause of the disease. In addition to a paediatric ophthalmological and neurological examination, many patients may be subject to specialised investigations that include electroretinograms (ERGs), visual evoked potentials (VEPs), optical coherence tomography (OCT) and, in some instances, MRI under anaesthesia/sedation for children who cannot cooperate. Previous work has shown that in approximately $40 \%$ of cases of IN VEPs were inconclusive in children due to unreliable traces. ${ }^{10}$ Obtaining reliable results from other investigative modalities, such as OCT, are also user-dependent and only available in a few centres for infants and young children. It also requires significant co-operation from the patient, which can be challenging in children.

With the advent of next-generation sequencing (NGS) it is now possible to develop targeted sequencing panels for a specific phenotype. However, to date there have been no studies systematically investigating the use of a targeted NGS panel for IN. Therefore, we aimed to develop a NGS panel to investigate its use in clinical practice, specifically assessing sensitivity, specificity and diagnostic potential of this panel.

\section{MATERIALS AND METHODS}

Subjects

Fifteen patients with IN were recruited from the University Hospitals of Leicester to take part in this study. Patients were chosen randomly from a

\footnotetext{
${ }^{1}$ Ulverscroft Eye Unit, Department of Neuroscience, Psychology and Behaviour, University of Leicester, Leicester, UK

${ }^{2}$ These authors contributed equally to this work.

${ }^{*}$ Correspondence: Dr MG Thomas or Professor I Gottlob, Ulverscroft Eye Unit, Department of Neuroscience, Psychology and Behaviour, University of Leicester, RKCSB, PO Box 65, Leicester, Leicestershire LE2 7LX, UK. Tel: +44 116252 3152; Fax: +44 116223 1996; E-mail: mt350@le.ac.uk or ig15@le.ac.uk

Received 4 September 2016; revised 20 February 2017; accepted 1 March 2017; published online 5 April 2017
} 
database $^{1}$ of 300 familial cases of IN. The inclusion criteria for this study were (a) onset of nystagmus within the first 6 months of life and (b) clinical suspicion of a Mendelian inheritance. Previous work in idiopathic IN (IIN) singletons identified FRMD7 variants between 0 and $7 \%$ of cases, thus suggesting that novel genes or non-genetic factors could be causative in singletons. ${ }^{11,12}$ Exclusion criteria were acquired nystagmus or evidence of contributory non-genetic aetiological factors for the nystagmus such as retinopathy of prematurity, infections, trauma, drug exposure (eg, opiates, benzodiazepines) in utero and fusional maldevelopment nystagmus syndrome. All patients underwent detailed ophthalmic examinations. Eye movement recordings, electrodiagnostic tests and OCT were also obtained where possible. Eye movement recordings were obtained using infrared video pupil tracker (EyeLink II and EyeLink1000, SR Research Ltd, Ontario, Canada). OCTs were obtained using handheld OCT (Bioptigen Envisu system, Durham, NC, USA) and a table-mounted device (SOCT Copernicus HR, OPTOPOL Technology S. A., Zawiercie, Poland). Each clinical diagnosis was based on specialist paediatric ophthalmologist assessment (by IG) and determined prior to sequence analysis. Authors MGT and GDEM performed the sequence analysis and therefore were blinded to the clinical diagnosis and phenotypical characteristics. The clinical characteristics of the patients are shown in Table 1. Written consent was obtained from all patients (or parents in case of children unable to consent). The study adhered to the tenets of the Declaration of Helsinki and was approved by the local ethics committee.

\section{Nystagmus gene panel development and NGS}

Saliva samples were obtained from all patients using the Oragene DNA sample Collection Kit (OG-500, DNA Genotek Inc., Ottawa, Ontario, Canada). DNA was extracted using the Qiagen DNA extraction kits as per the manufacturer's recommendations.

The genomic DNA was randomly fragmented with a base pair peak of 200$250 \mathrm{bp}$, subsequently adapters were ligated to both ends of the resulting fragments. DNA was amplified by ligation-mediated PCR, purified and hybridised to NimbleGen Human custom array (NimbleGen SeqCap EZ Choice, Roche Nimblegen Inc., Madison, WI, USA) for enrichment. NimbleDesign was used to create the custom probeset design for the nystagmus panel. All known genes associated with IN were included within the panel $(n=336)$. The genes were selected based on previous literature, OMIM records (www.omim.org) and RetNet, the Retinal Information Network ${ }^{13}$ (see Supplementary Table S1). The probes were designed to capture the exons and 40 bases flanking the splice junction. If there were more than one transcript, we chose the longest transcript from the GRCh37/hg19 assembly. We also included known cis-regulatory elements associated with nystagmus genes that have previously been described in the literature. ${ }^{14,15}$ Specific targets included: (1) an ultraconserved cis-element located $150 \mathrm{~kb}$ downstream from $P A X 6$, variants of which has been described in a patient with aniridia and nystagmus (variant: hg19: chr11:g.31685945G $>$ T) ${ }^{15}$ and (2) highly conserved cis-elements located up to $11 \mathrm{~kb}$ upstream of the TYR gene as described by Ray et $a l,{ }^{14}$ which includes the TYR promoter. This region is hypothesised to harbour variants causative of albinism ${ }^{14}$; however, to date no sequencing study has been undertaken to include this region.

Resulting libraries were sequenced on a HiSeq 2000 (Illumina, San Diego, CA, USA) according to the manufacturer's recommendations for paired-end $(2 \times 100 \mathrm{bp})$ protocol. The mean coverage depth ranged between $181 \times$ and $301 \times$ (Supplementary Table S2). Percentage of the target sequence covered $>15 \times$ was $>99 \%$ (Supplementary Table S2).

All 15 IN samples and the reference DNA (NA12878-see below) were enriched and sequenced as described above. Allelic variants were reported according to Human Genome Variation Society guidelines. ${ }^{16}$ We identified rare variants by focussing on protein-altering and splice-site changes with an allele frequency of $<1 \%$ in the 1000 genomes project or in the NHLBI ESP exomes. Variants that were previously established to cause IN were included and classified as pathogenic even if allele frequency was $>1 \%$. The allelic variations were also assessed against the sequence data from 70 controls (without nystagmus) and were excluded if present within our control data set. The control cohort consisted of a mixture of ethnicities: European $(n=52)$, South Asian $(n=13)$, and East Asian $(n=5)$.
The variant and phenotype data have been submitted to the Leiden Open Variation Database (http://databases.lovd.nl/shared; individual IDs 0008817600088188).

Nystagmus gene panel validation experiment and sequence analysis The National Institute of Standards and Technology together with the Genome in a Bottle Consortium have developed reference genome material (NA12878 DNA and high-confidence variant calls), which is recommended for assessing variant-call accuracy and understanding biases when developing novel targeted sequencing panels. This will not only consider the efficiency of the capture and sequencing process but also provide a benchmark against which we could assess different alignment and variant-calling algorithms. We obtained the NA12878 DNA (reference DNA) from Coriell Cell repositories. Library construction and sequencing was performed as detailed above.

The read files obtained from sequencing were analysed using the BWAGATK pipeline. The variants generated were assessed against previously published gold-standard variants for the sample. ${ }^{17}$ This includes a combination of 11 NA12878 whole-human genome data sets and three exome data sets, generated across five sequencing platforms to eliminate bias from any single platform. The high-confidence variant calls are available at: $\mathrm{ftp} / / / \mathrm{ftp}$-trace.ncbi. nlm.nih.gov/giab/ftp/release/NA12878_HG001/.

The reads were aligned using BWA v0.7.5 ${ }^{18}$ to the reference genome (hg19). Samtools v1.2 ${ }^{19}$ was used to convert, sort and index the alignment.bam files. Picard v1.93 (http://broadinstitute.github.io/picard/) was used to remove duplicates. GATK v3.4-0 ${ }^{20}$ was used for local realignment around indels and recalibrate quality scores. Single-nucleotide variants and indels were detected using GATK. Further annotation and filtering was performed using ANNOVAR. ${ }^{21}$ We utilised FishingCNV v2.1 for copy number variation analyses. ${ }^{22}$

We determined the assay sensitivity, specificity and balanced accuracy for the NA12878 sample by computing the true positives (TP), false positives (FP), true negatives $(\mathrm{TN})$ and false negatives $(\mathrm{FN})$. Sensitivity $=\mathrm{TP} /(\mathrm{TP}+\mathrm{FN})$; specificity $=$ $\mathrm{TN} /(\mathrm{TN}+\mathrm{FP})$ and balanced accuracy $=($ sensitivity + specificity $) / 2$.

Sequence analyses were performed on a high-performance computing cluster at the University of Leicester.

\section{RESULTS}

\section{Diagnostic potential of the nystagmus gene panel}

The pedigrees of all the 15 subjects associated with IN are shown in Supplementary Figure S1. The phenotypical characteristics and investigations performed are summarised in Table 1. The significant variants identified are shown in Table 2. Examples of nystagmus waveforms identified on eye movement recordings are shown in Supplementary Figure S2. This shows significant familial variability of nystagmus waveforms.

The masked analyses revealed pathogenic or probable pathogenic variants in 9 out of the 15 patients (Table 2). This was consistent with the clinical diagnosis in six out of the nine patients. A hemizygous missense (c. 796G > C, p.(Ala266Pro)) and frameshift (c.1262delC, p.(Pro421LeufsTer23)) variants of FRMD7 were identified in NYS-001 and NYS-003, respectively. The clinical features were consistent with a diagnosis of IIN. Both patients had relatively good visual acuity, stereopsis and horizontal nystagmus. In NYS-003 ERGs, VEPs and OCTs were normal. It was not possible to perform further investigations in NYS-001 due to poor cooperation. Hemizygous missense (c.299T > G, p.(Leu100Arg)) and nonsense (c.2905C> T, p.(Arg969Ter)) variants of CACNA1F were identified in NYS-002 and NYS-004, respectively; both patients were male subjects with reduced visual acuity and predominantly horizontal nystagmus with a vertical component. ERGs showed the characteristic 'negative' waveform (a-wave larger than the b-wave) in response to bright flashes after dark adaptation (scotopic conditions), consistent with the clinical diagnosis of congenital stationary night blindness. Significant 


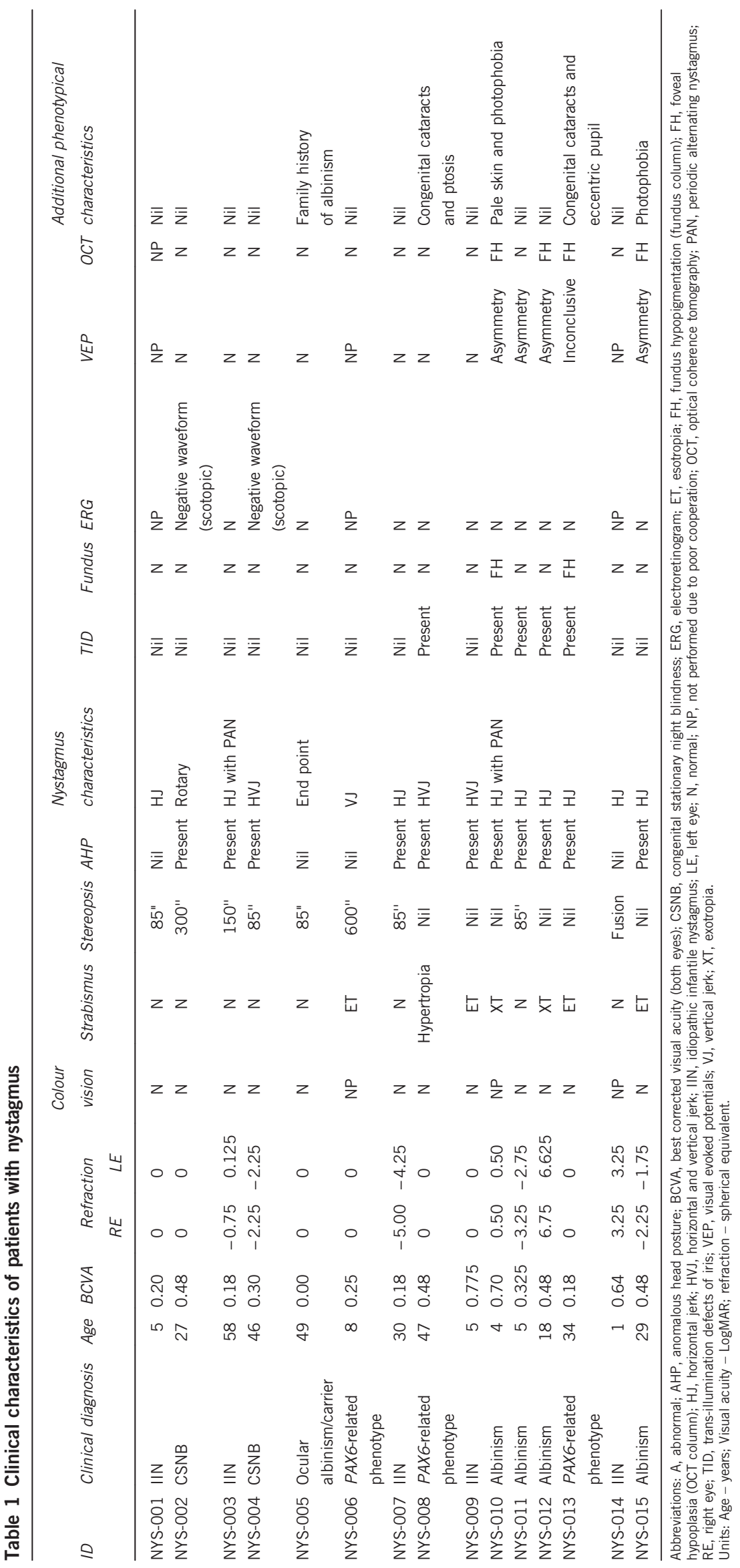




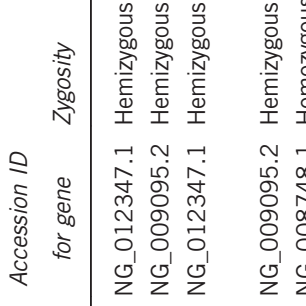

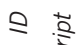

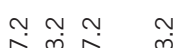

离



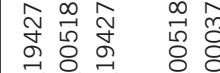

$\sum^{1} \sum_{i} \sum_{\Sigma} \sum_{z}$

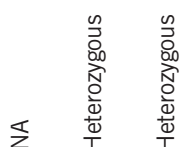

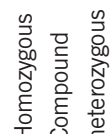





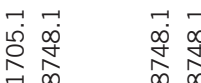



$\vec{\infty}$

矛

a $m \underset{\sim}{\sim}$

管

$\begin{array}{ll}1 & 0 \\ -1 & 8 \\ 0 & 8\end{array}$

o

o

๑ั

员

ㄴํㅇ ช

$\sum_{i} \sum$

ए

空 $\stackrel{0}{4}$

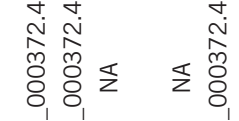

$\sum_{2}$

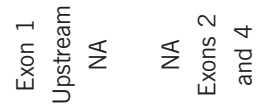



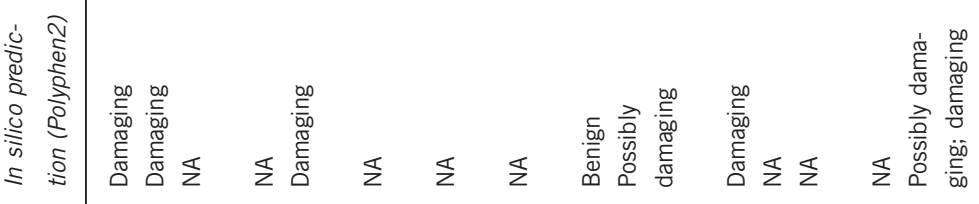

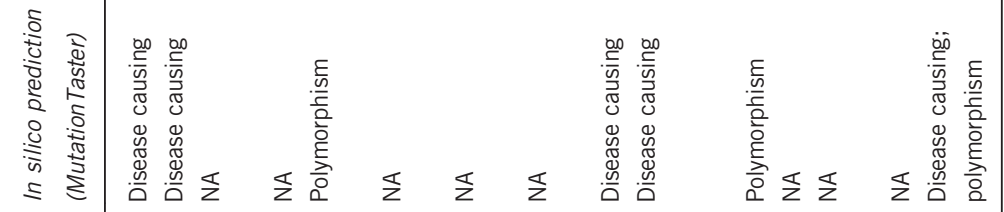

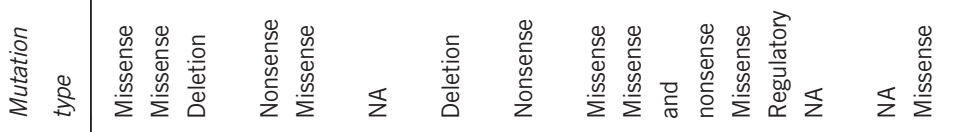




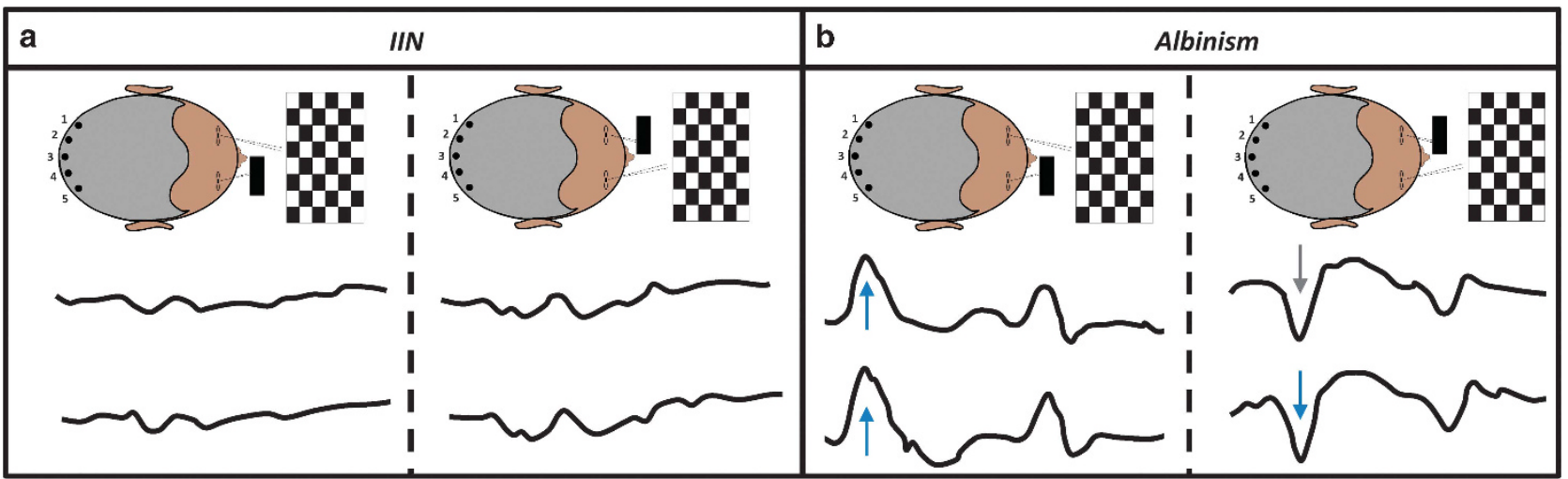

C
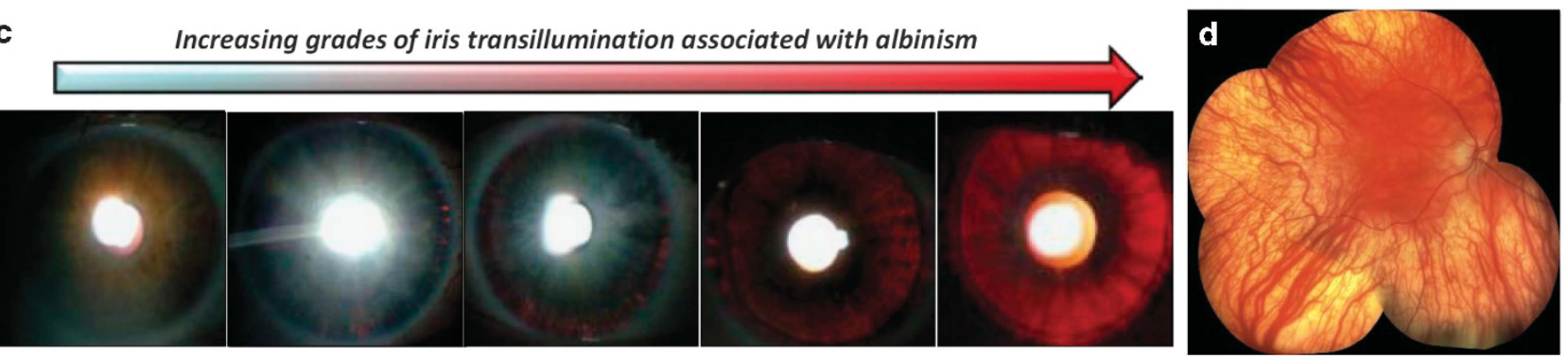

Figure 1 Multichannel VEPs to investigate intracranial visual pathway dysfunction (a and $\mathbf{b}$ ). Monocular stimulation is achieved by occluding one eye and presenting a pattern stimulus (checkerboard stimulus). Four lateral electrodes ( 1 and 2 on left occiput, 4 and 5 on the right occiput) and one midline electrode (electrode 3) are used. Five raw traces corresponding to the polarity of each electrode are obtained during monocular stimulation. In order to improve signal to noise, VEP traces shown are based on the polarity differences between the electrodes placed on the left and right occiput and obtained after averaging. Individuals with a normal visual pathway would not have any significant difference in the polarity between the corresponding lateral electrodes ( 1 vs 5 or 2 vs 4). Thus a subtracted waveform (1-5 and 2-4) would show no significant polarity differences as seen in the IIN patient (NYS-007) (a). However, in a patient with chiasmal misrouting as seen in albinism (NYS-012) asymmetric responses are seen (blue arrow) (b). Different degrees of iris transillumination defects seen with albinism (c). Pale fundus associated with albinism (d).

intrafamilial variability of nystagmus was noted in NYS-002 (Supplementary Figure S2).

Compound heterozygous variants of $T Y R$ were identified in NYS-010 (c.1A > G, p.(Met1Val) and c.346C > T, p. (Arg116Ter)) and NYS-015 (c.823G > T, p.(Val275Phe) and c.1205G > A, p.(Arg402Gln)). Both patients had significantly reduced visual acuity, no detectable stereopsis, hypopigmentation of the skin, trans-illumination defects of the iris, horizontal nystagmus and foveal hypoplasia. Multichannel VEPs showed asymmetry of hemispheric responses on monocular stimulation indicating chiasmal misrouting (Figure 1). The phenotypical features were consistent with a clinical diagnosis of albinism. The location of the amino-acid changes in relation to the protein are shown in Supplementary Figure S3. Foveal hypoplasia identified using OCT is shown in Figure 2. Significant intrafamilial variability in foveal morphology was noted in family NYS-011.

In three out of the nine patients, the genetic diagnosis allowed us to resolve the clinical diagnosis. The clinical diagnosis in NYS-005 was unclear as the phenotype was very mild with good visual acuity, end point nystagmus, no iris trans-illumination defects, normal electrodiagnostic studies and normal retinal morphology on OCT. However, there was a family history of albinism and therefore the working clinical diagnoses included ocular albinism or a carrier for albinism. The eye movement recordings showed variable nystagmus in the family with subjects III:1 and III:2 having pseudopendular nystagmus with foveating saccades while subject II:2 did not have any nystagmus on central fixation but had square wave jerks (Supplementary Figure S2). We identified a homozygous missense variant (c.1205G > A, p.(Arg402Gln)) in TYR. In NYS-008, a possible $P A X 6$-related phenotype was considered as the clinical diagnosis based on the history of congenital cataracts, ptosis, trans-illumination defects of the iris, reduced visual acuity and nystagmus. Sutural cataracts seen as the opacification of the Y-suture of the fetal nucleus were identified in both of his daughters (Figure 3). Interestingly, two variants were identified: the first was a novel heterozygous variant in CRYBA1 (c.594G > A, p.(Trp198Ter)), and the second was a novel heterozygous variant in COL11A1 (c. 2956C >A, p.(Pro986Thr). There was no myopia or history of retinal detachment, suggesting the variant in CRYBA1 was causative of the phenotype. In NYS-009, the clinical diagnosis of IIN was established based on normal iris, electrodiagnostic tests and OCTs but visual acuity was lower than expected. However, sequence analysis revealed a homozygous missense (c.1579G $>$ C, p.(Glu527Gln)) variant in TYRP1 consistent with albinism.

Initially, in 6 out of the 15 patients, we did not identify the causative variant based on the masked analysis. However, unmasked to the clinical information we re-analysed the sequence data. In NYS-007, the phenotype was consistent with IIN with an X-linked pedigree. The copy number variation analysis performed using FishingCNV revealed a heterozygous deletion of the FRMD7 gene. Multiplex Ligationdependent Probe Amplification (MLPA) analysis showed a large deletion of FRMD7 exons 2-12. In NYS-011, a previously filtered variant (c.575C > A, p.(Ser192Tyr)) was identified in TYR. The variant was filtered out owing to the preset threshold of allele frequency $<1 \%$. The exact pathogenicity of the variant is yet to be determined, 

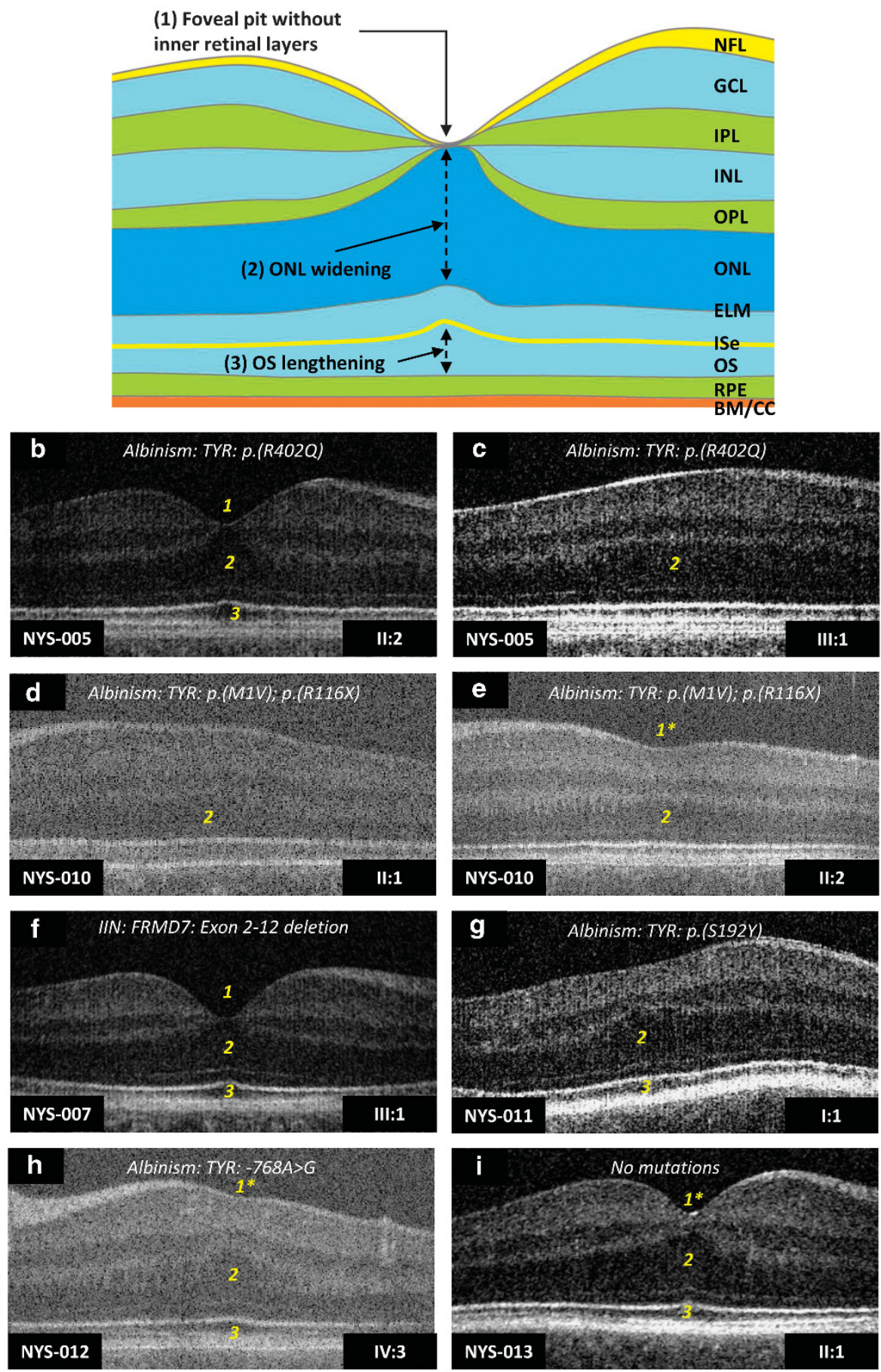

Figure 2 Optical coherence tomograms showing the retinal structure in patients with IN. Figure (a) shows an illustration of normal foveal morphology with the features that indicate foveal specialisation which include: (1) deep foveal pit, (2) displacement of inner retinal layers, (3) ONL widening, and (4) OS lengthening. In family NYS-005, the mother (II:2) had a normal foveal structure (b) with all four features present (labelled 1-4); however, her son (III:1) did not have a foveal pit and only had ONL widening (c). Similarly, in family NYS-010 we observe phenotypical heterogeneity with regards to the foveal structure. Both sisters had compound heterozygous variants of the TYR gene; however, in subject II:1 there was only ONL widening, whereas in subject II:2 there was both ONL widening and a rudimentary foveal pit (denoted by $1 *$ ). In family NYS-007, OCT from subject III:1 with FRMD7 deletions has normal foveal morphology. Subject I:1 in family NYS-011 with albinism has no foveal pit but has ONL widening and OS lengthening. In family NYS-012 with albinism, we observe foveal hypoplasia with a rudimentary foveal pit $\left(1^{*}\right)$ in subject IV:3, as well as ONL widening and OS lengthening. In family NYS-013, we were unable to identify any variants that affect function within the known nystagmus genes; however, subject II: 1 was noted to have subtle foveal hypoplasia with a shallow foveal pit $\left(1^{*}\right)$ and persistence of inner retinal layers posterior to the fovea. The numbers (1-4) within the tomograms represents the presence of the foveal specialisation, with 1 * representing a shallow pit in foveal hypoplasia. Abbreviations: NFL, nerve fibre layer; GCL, ganglion cell layer; IPL, inner plexiform layer; INL, inner nuclear layer; OPL, outer plexiform layer; ONL, outer nuclear layer; ELM, external limiting membrane; ISe, inner segment ellipsoid; OS, outer segment; RPE, retinal pigment epithelium; BM/CC, Bruchs membrane/choriocapillaries. 

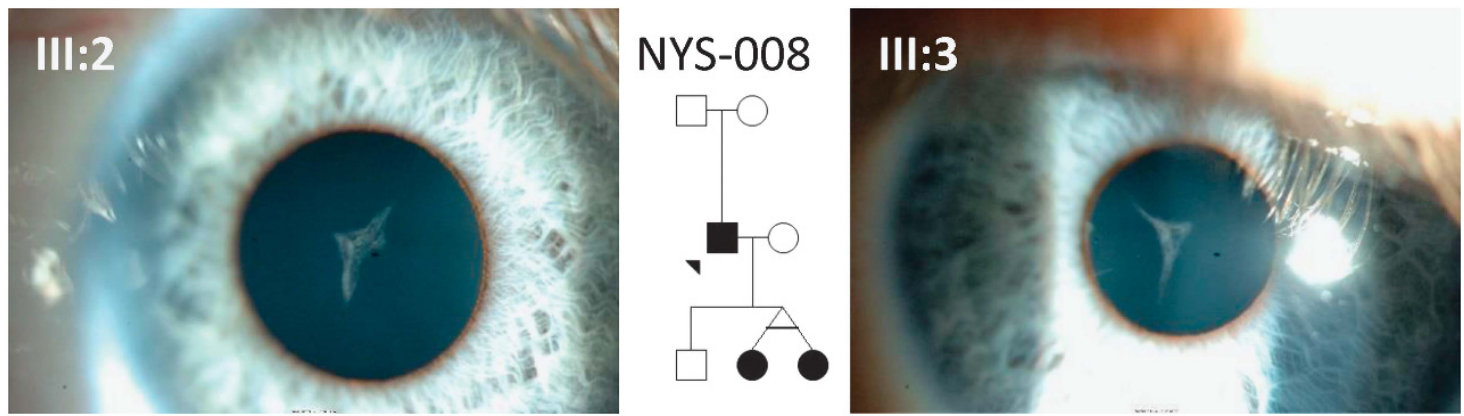

Figure 3 Congenital cataracts associated with CRYBA1 variant in NYS-008. The two images from subjects III:2 and III:3 show (monozygotic twins) sutural cataracts, seen as the opacification of the Y-suture of the foetal nucleus of the lens. Subject II:1 was pseudophakic.

but there is some evidence that there is reduced enzymatic activity of tyrosine hydroxylase and DOPA oxidase when the Tyr192 variant allele was compared with the Ser192 wild-type allele. ${ }^{23}$ In NYS-012, we identified a homozygous genomic variant (hg19 chr11:g. $88910354 \mathrm{~A}>\mathrm{G})$ upstream from the transcription start site for the TYR gene (c.-768A $>$ G; Supplementary Figure S3). This was a rare variant that has previously not been reported in the literature or in control databases (1000 genomes project, NHLBI ESP exomes or Exomes Aggregation Consortium (ExAC)). We suspect this is likely to affect function as it is located within a GA complex repeat region hypothesised to regulate the transcriptional efficiency of TYR. ${ }^{14}$ In the remaining 3 out of 15 patients (NYS-006, NYS-013 and NYS-014), we were not able to identify a variant responsible for the phenotype.

\section{Nystagmus gene panel validation experiment}

The variants identified following sequencing the NA12878 sample were compared against the previously published gold-standard variants for the sample to determine sensitivity and specificity. We identified a total of $1362 \mathrm{TP}$. This represents the called variants with a corresponding position in the reference variant file (VCF). A total of $511 \mathrm{FP}$ were identified that represents the called variants without a corresponding position in the reference VCF. A total of 2241377 TN were identified. This represents the called reference bases without a corresponding position in the reference VCF. A total of $20 \mathrm{FN}$ were identified. This represents the called reference bases with a corresponding position in the reference VCF. The validation experiment shows a sensitivity of $98.5 \%$ and a specificity of $99.9 \%$. The balanced accuracy from this study was $99.2 \%$.

\section{DISCUSSION}

We describe, for the first time, the clinical utility of a NGS panel for childhood nystagmus. This was achieved by designing a customised target enrichment kit for all known genes associated with IN. We analysed 15 patients with IN to assess the ability of this testing strategy in precisely determining the genetic cause of this disorder. We also tested one control subject (NA12878) to determine the assay sensitivity and specificity. During the masked analysis, we had a significant detection rate ( 9 out of the 15 patients) of variants definitely or probably responsible for the phenotype. The detection rate improved further (12 out of the 15 patients) with the clinical information and a targeted re-analysis of relevant genes. This highlights that even with masked analysis we can identify the genetic cause of most forms of IN. Among the 12 patients, we report two TYR variants (c.-768A $>\mathrm{G}$ and c. $575 \mathrm{C}>\mathrm{A}$ ) that requires further study. In the validation experiment, we show high levels of assay sensitivity (98.5\%), specificity (99.9\%) and balanced accuracy (99.2\%).
Though cost and access to screening is frequently an issue, NGS has replaced traditional Sanger sequencing in many clinical settings as the frontline diagnostic tool even prior to performing investigations. ${ }^{24}$ This allows for prompt and accurate diagnosis followed by relevant surgical or medical management. For genetically heterogeneous disorders, such as IN, the use of NGS is more cost effective than Sanger sequencing. It also has the potential to obviate the use of other investigations such as electrodiagnostic testing and neuroimaging, therefore reaching an accurate diagnosis coupled with appropriate genetic information relevant not only to the proband but also to other family members. In children with poor cooperation, electrodiagnostic testing can be unreliable and difficult to perform. ${ }^{10}$ Some investigations are also only available in specialist centres. The results from our blinded analyses from this study indicates that NGS has a role as a frontline diagnostic tool and therefore transforming the diagnostic pathway for patients with IN (Figure 4). It is important to emphasise that NGS is not a replacement for good clinical work up. Therefore, certain investigations would still be relevant based on the clinical context. For example, OCT in foveal hypoplasia also helps provide visual prognostic information based on the grade of foveal hypoplasia. ${ }^{6}$ Similarly, serial eye movement recordings might be necessary to provide an objective measurement of therapeutic response to medical or surgical intervention.

One of the drawbacks of NGS analysis is difficulty detecting copy number variations, yet we demonstrate in this study that we could detect a large FRMD7 deletion in a family with X-linked IIN where the initial analysis failed to identify this heterozygous deletion. During the masked analysis, the authors did not perform a CNV analysis; however, based on the strong clinical suspicion of X-linked IIN the authors performed a CNV analysis on the NGS data using FishingCNV v2.1, ${ }^{22}$ which revealed a large FRMD7 deletion. This was confirmed using MLPA analysis. No further CNVs were identified in the other families.

In three patients (NYS-005, NYS-008 and NYS-009), the genetic diagnosis helped resolve the clinical diagnosis. In patient NYS-005, we identified a variant (rs1126809) in TYR resulting in the amino-acid substitution p.(Arg402Gln). There has been some debate regarding the pathogenicity of this variant. Oetting et al ${ }^{25}$ reported that the R402Q variant is not associated with autosomal-recessive ocular albinism but postulated that a causative variant might be in genetic disequilibrium with the p.(Arg402Gln) variant. However, Chiang et al ${ }^{26}$ showed that this allele is strongly associated with albinism patients who have only 1 variant in TYR. Berson et al ${ }^{27}$ showed that the p. (Arg402Gln) variant resulted in a defect in protein folding, which prevents exit from the endoplasmic reticulum thus resulting in an abnormal phenotype. In patient NYS-009, with clinical diagnosis of IIN but with genetic 
Nystagmus Diagnostic Workflow

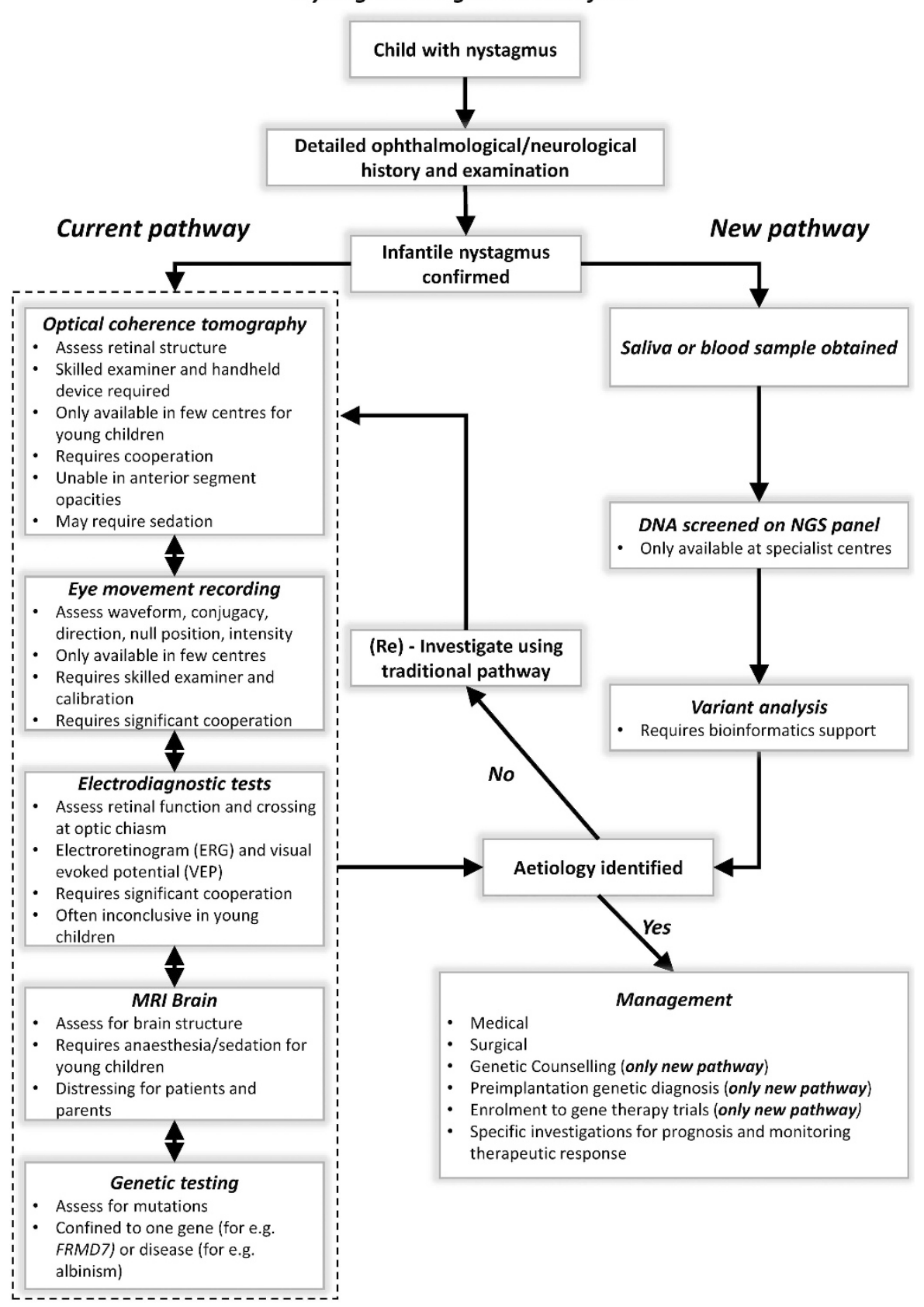

Figure 4 Diagnostic workflow for patients with nystagmus. In the current pathway, patients would undergo a number of different investigations prior to being able to identify the underlying aetiology. The order of performing investigations vary between centres, depends on the clinical context, patient cooperation and availability. Some of the limitations associated with each investigative modality is shown in the box. Not all patients would undergo MRI scans; however, it is one of the investigations of choice for specific forms of nystagmus (for eg, vertical nystagmus) or the presence of additional neurological features such as ataxia were noted. The new pathway that involves using NGS as a frontline diagnostic tool would help establish a genetic diagnosis and thus guide further investigations and targeted treatment.

diagnosis of TYRP1, interestingly we noted both horizontal and vertical nystagmus. Previously, a small vertical component to the nystagmus has been described with both FRMD7 variants and albinism. ${ }^{28}$ However, in this patient the amplitude of the vertical and horizontal components was similar that was atypical.

Patient NYS-008 was originally thought to have a PAX6-related phenotype; however, we identified two novel variants in CRYBA1 and COL11A1 resulting in the amino-acid substitution p.(Trp198Ter)and
p.(Pro986Thr), respectively. Both variants segregated with the phenotype. This highlights the complexity in formulating a diagnosis in patients with IN. Although the patient had congenital cataract, he also had additional features that included ptosis, trans-illumination defects of the iris, reduced visual acuity and nystagmus, therefore a diagnosis of PAX6-related phenotype was considered. COL11A1 variants have been described in association with Stickler/Marshall syndrome. ${ }^{29,30}$ The patient did not have the classic features of Stickler/Marshall 
syndrome such as high myopia, retinal detachment and mid facial hypoplasia. Previously, the amino-acid substitution p.(Gly988Val) in COL11A1 has been described ${ }^{29,30}$; this is very close to the variant p.(Pro986Thr) that we have reported. Interestingly, the phenotype with the G988V variant was also milder, not associated with retinal detachments; however, cataract and high myopia were present. Nystagmus has previously been described to be associated with COL11A1 variant. ${ }^{31}$ However, as there was a truncating variant of CRYBA1 we suspect this is most likely the cause of the phenotype. Previously, nystagmus has been described to be associated with congenital cataracts due to CRYBA1 variants. ${ }^{32}$ However, the additional phenotypical features such as ptosis and iris transillumination defects have not been documented.

To date, there have been no comparisons between diagnostic ability of exome sequencing and targeted NGS panels in nystagmus. The main advantages of the targeted NGS panel over exome sequencing are: (1) cheaper cost and (2) ability to sequence genomic regions not included or poorly covered within the exome target regions. One of the unique features of the nystagmus NGS panel is that it not only captures the coding sequence along with 40 bases flanking the splice junction but also cis-regulatory elements associated with nystagmus genes and deep intronic regions that have previously been described in the literature. ${ }^{7,14,15}$ These are genomic regions that could potentially be missed during whole-exome sequencing. For example, previously we have identified deep intronic variants in the FRMD7 gene (c.285-118C > T) predicted to activate a cryptic splice donor ${ }^{7}$; using an exome sequencing approach, we would have missed these variants. However, in the panel described here we have included these additional sites associated with IN. Similarly, we included regulatory sites upstream from the TYR gene. This has allowed us to identify a novel variant upstream from the transcription start site for TYR within the GA complex repeat region hypothesised to regulate transcriptional efficiency. ${ }^{14}$ The importance of clinical context was highlighted in subject NYS-011; in our masked analysis, we did not identify any variants likely to affect function. However, her phenotype consisted of (1) asymmetric VEPs suggestive of abnormal decussation of axons at the optic chiasm and (2) trans-illumination defects of the iris, both features of albinism. We, therefore, reviewed all variants within the albinism-related genes and genes associated with optic nerve misrouting. ${ }^{8}$ We identified a previously filtered homozygous TYR variant resulting in the amino-acid substitution p.(Ser192Tyr). The variant was filtered out owing to our preset threshold of filtering variants with a minor allele frequency $>1 \%$. Previous work has suggested that this variant is associated with variation in skin pigmentation $^{33}$; however, the effects of a homozygous variant is unclear. The Tyr192 variant allele was associated with reduced enzymatic activity of tyrosine hydroxylase and DOPA oxidase when compared with the Ser192 wild-type allele. ${ }^{23}$ Further functional work is necessary to understand the exact effects of both these variants on transcription and translation of TYR.

In three patients (NYS-006, NYS-013, NYS-014), we did not identify any significant variants within all known nystagmus-related genes. This is unlikely to be due to insufficient depth of coverage as all three samples had an average coverage depth $>180 \times$. It is possible that these families harbour variants within novel genes associated with nystagmus or within other cis-regulatory elements that are yet to be identified. Therefore, in these families, it would be appropriate to perform whole-genome sequencing to identify the genetic basis of their nystagmus. This study has also highlighted several atypical features that can be encountered such as vertical nystagmus (NYS-009) and a normal retinal structure on OCT (NYS-011) associated with albinism. Hence, the phenotypic spectrum associated with these disorders is larger than we had previously thought, and as NGS testing becomes more accessible, we will be able to identify more genotype-phenotype correlations.

In conclusion, this study shows that NGS has an important role in early screening and diagnosis of patients with IN. It has the potential to complement other investigations currently used for diagnostic purposes and reveal atypical phenotypes associated with certain genes. We demonstrate that the genetic diagnosis was used to revise the clinical diagnosis. Thus it will have a role in the development of an individualised care plan and identifying the genetic basis of phenotypes with atypical features. Moreover, the genetic information enables the accurate genetic counselling and further family planning. With the advent of personalised genomic medicine, the use of the nystagmus NGS panel is a cost-effective frontline diagnostic tool.

\section{CONFLICT OF INTEREST}

The authors declare no conflict of interest.

\section{ACKNOWLEDGEMENTS}

This study was supported by the Ulverscroft Foundation, Fight for Sight (grant ref: 5009/5010) and the Medical Research Council (MRC), London, UK (grant number: MR/J004189/1 and MRC/N004566/1). MGT is supported by the NIHR (no. 2980). This research used the ALICE High Performance Computing Facility at the University of Leicester. We thank the assistance of Mr Ravi Purohit, Dr Shafak Toufeeq and Dr Zhanhan Tu with eye movement recordings, fundus photography and electrodiagnostics.

1 Sarvananthan N, Surendran M, Roberts EO et al: The prevalence of nystagmus: the Leicestershire nystagmus survey. Invest Ophthalmol Vis Sci 2009; 50: 5201-5206.

2 Pilling RF, Thompson JR, Gottlob I: Social and visual function in nystagmus. $\mathrm{Br} J$ Ophthalmol 2005; 89: 1278-1281.

3 McLean RJ, Windridge KC, Gottlob I: Living with nystagmus: a qualitative study. $\mathrm{Br} \mathrm{J}$ Ophthalmol 2012; 96: 981-986.

4 Michaelides M, Hunt DM, Moore AT: The cone dysfunction syndromes. Br J Ophthalmol 2004; 88: 291-297.

5 Gottlob I: Nystagmus. Curr Opin Ophthalmol 2000; 11: 330-335.

6 Thomas MG, Kumar A, Mohammad S et al: Structural grading of foveal hypoplasia using spectral-domain optical coherence tomography a predictor of visual acuity? Ophthalmology 2011; 118: 1653-1660.

7 Thomas MG, Crosier M, Lindsay S et al: Abnormal retinal development associated with FRMD7 mutations. Hum Mol Genet 2014; 23: 4086-4093.

8 Poulter JA, Al-Araimi M, Conte I et al: Recessive mutations in SLC38A8 cause foveal hypoplasia and optic nerve misrouting without albinism. Am J Hum Genet 2013; 93: 1143-1150.

9 Leigh RJ, Khanna S: What can acquired nystagmus tell us about congenital forms of nystagmus? Semin Ophthalmol 2006; 21: 83-86.

10 Lee H, Sheth V, Bibi M et al: Potential of handheld optical coherence tomography to determine cause of infantile nystagmus in children by using foveal morphology. Ophthalmology 2013; 120: 2714-2724.

11 Tarpey P, Thomas S, Sarvananthan N et al: Mutations in FRMD7, a newly identified member of the FERM family, cause X-linked idiopathic congenital nystagmus. Nat Genet 2006; 38: 1242-1244.

12 Schorderet DF, Tiab L, Gaillard MC et al: Novel mutations in FRMD7 in X-linked congenital nystagmus. Mutation in brief \#963. Online. Hum Mutat 2007; 28: 525 .

13 Daiger S, Rossiter B, Greenberg J, Christoffels A, Hide W: Data services and software for identifying genes and mutations causing retinal degeneration. Invest Ophthalmol Vis Sci 1998; 39: S295.

14 Ray K, Chaki M, Sengupta M: Tyrosinase and ocular diseases: some novel thoughts on the molecular basis of oculocutaneous albinism type 1. Prog Retin Eye Res 2007; 26: 323-358.

15 Bhatia S, Bengani H, Fish M et al: Disruption of autoregulatory feedback by a mutation in a remote, ultraconserved PAX6 enhancer causes aniridia. Am J Hum Genet 2013; 93: 1126-1134

16 den Dunnen JT, Antonarakis SE: Mutation nomenclature extensions and suggestions to describe complex mutations: a discussion. Hum Mutat 2000; 15: 7-12.

17 Zook JM, Chapman B, Wang J et al: Integrating human sequence data sets provides a resource of benchmark SNP and indel genotype calls. Nat Biotechnol 2014; 32 246-251. 
$18 \mathrm{Li} \mathrm{H}$, Durbin R: Fast and accurate long-read alignment with Burrows-Wheeler transform. Bioinformatics 2010; 26: 589-595.

$19 \mathrm{Li} \mathrm{H}$, Handsaker B, Wysoker A et al: The Sequence Alignment/Map format and SAMtools. Bioinformatics 2009; 25: 2078-2079.

20 McKenna A, Hanna M, Banks E et al: The Genome Analysis Toolkit: a MapReduce framework for analyzing next-generation DNA sequencing data. Genome Res 2010; 20: 1297-1303.

21 Wang K, Li M, Hakonarson H: ANNOVAR: functional annotation of genetic variants from high-throughput sequencing data. Nucleic Acids Res 2010; 38: e164.

22 Shi Y, Majewski J: FishingCNV: a graphical software package for detecting rare copy number variations in exome-sequencing data. Bioinformatics 2013; 29: 1461-1462.

23 Chaki M, Sengupta M, Mondal M et al: Molecular and functional studies of tyrosinase variants among Indian oculocutaneous albinism type 1 patients. J Invest Dermatol 2011; 131: 260-262.

24 Gillespie RL, O'Sullivan J, Ashworth J et al: Personalized diagnosis and management of congenital cataract by next-generation sequencing. Ophthalmology 2014; 121: 2124-37.e1-2.

25 Oetting WS, Pietsch J, Brott MJ et al: The R402Q tyrosinase variant does not cause autosomal recessive ocular albinism. Am J Med Genet A 2009; 149A: 466-469.

26 Chiang PW, Spector E, Tsai AC: Oculocutaneous albinism spectrum. Am J Med Genet A 2009; 149A: 1590-1591.

27 Berson JF, Frank DW, Calvo PA, Bieler BM, Marks MS: A common temperaturesensitive allelic form of human tyrosinase is retained in the endoplasmic reticulum at the nonpermissive temperature. J Biol Chem 2000; 275: 12281-12289.

28 Kumar A, Gottlob I, Mclean RJ, Thomas S, Thomas MG, Proudlock FA: Clinical and oculomotor characteristics of albinism compared to FRMD7 associated infantile nystagmus. Invest Ophthalmol Visual Sci 2011; 52: 2306-2313.
29 Annunen S, Korkko J, Czarny $M$ et al: Splicing mutations of 54-bp exons in the COL11A1 gene cause Marshall syndrome, but other mutations cause overlapping Marshall/Stickler phenotypes. Am J Hum Genet 1999; 65: 974-983.

30 Zlotogora J, Sagi M, Schuper A, Leiba H, Merin S: Variability of Stickler syndrome. Am J Med Genet 1992; 42: 337-339.

31 Griffith AJ, Gebarski SS, Shepard NT, Kileny PR: Audiovestibular phenotype associated with a COL11A1 mutation in Marshall syndrome. Arch Otolaryngol Head Neck Surg 2000; 126: 891-894.

$32 \mathrm{Lu} \mathrm{S}$, Zhao C, Jiao $\mathrm{H}$ et al: Two Chinese families with pulverulent congenital cataracts and deltaG91 CRYBA1 mutations. Mol Vis 2007; 13: 1154-1160.

33 Stokowski RP, Pant PV, Dadd T et al: A genomewide association study of skin pigmentation in a South Asian population. Am J Hum Genet 2007; 81: 1119-1132.

(c) (i) This work is licensed under a Creative Commons Attribution 4.0 International License. The images or other third party material in this article are included in the article's Creative Commons license, unless indicated otherwise in the credit line; if the material is not included under the Creative Commons license, users will need to obtain permission from the license holder to reproduce the material. To view a copy of this license, visit http:// creativecommons.org/licenses/by/4.0/

(C) The Author(s) 2017

Supplementary Information accompanies this paper on European Journal of Human Genetics website (http://www.nature.com/ejhg) 